\title{
Review of: "Inflammation induces pro-NETotic neutrophils via TNFR2 signaling"
}

\author{
Leslie Chavez-Galan ${ }^{1}$ \\ 1 Instituto Nacional de Enfermedades Respiratorias
}

Potential competing interests: The author(s) declared that no potential competing interests exist.

In this study, Neuenfeldt F et al. has the aim to analyse if there are PMN subpopulations with an altered functional repertoire or susceptibility to NETosis could explain increased NETosis in inflamed tissues.

From the view of this reviewer, this topic is excited and original, this topic is really interesting in a wide field of research, for instances in the study of inflammation, neutrophils function, TNF pathway, TNF retrosignalling, etc. Although this study has a well scientific background and authors showed strong evidence of their main I have some questions that I will appreciate if authors provide the answers in order to improve this interesting manuscript.

Mayor comments

1. How did you distinguish between intracellular and transmembrane TNF? because in Method section, authors mention only the use of one mAb anti-TNF with one fluorochrome (TNFCE士-PE-Dazzle594). You need 2 antibodies with different fluorochrome and in this way you can distinguish between intra and extra. Moreover, did you fix the cells to be sure that tmTNF is not suffering a quickly recirculation in the cells of PMN CCR5-?

2. I did not find how did you do the migration assay? It is not in method section, and I would like to know why the stimuli was 5 minutes. Have you a direct antecedent or did you do a time-curve?

3. In discussion section authors wrote that tmTNF only interacts with TNFR2 and TNFR1 only with sTNF and it is not absolute true, there are reports that showed the interaction of TNFR1 with tmTNF (Nat Neurosci 16 (2013) 865-73; Infect Immun 73 (2005) 3668-76.). Considering this data maybe rephrase some phrases in the discussion section.

4. Please provide the full name of the Bioethical Committee that authorised this project and the number project.

Minor comments 
1. Line 158, why you wrote "(SELL)" but after you did not use that abbreviation?, you still using CD62L. 2. Line 518 , remove "))" 